\title{
Taurine Administration Recovers Motor and Learning Deficits in an Angelman Syndrome Mouse Model
}

\author{
Sara Guzzetti ${ }^{1,+}{ }^{+}$, Luciano Calzari ${ }^{1,+}{ }^{+}$, Lucia Buccarello ${ }^{1}$, Valentina Cesari ${ }^{2}$, Ivan Toschi ${ }^{2}$ (D), \\ Stefania Cattaldo ${ }^{3}$, Alessandro Mauro ${ }^{3,4,5}$, Francesca Pregnolato ${ }^{6}$, Silvia Michela Mazzola ${ }^{7}$ (i) \\ and Silvia Russo ${ }^{1, *}$ \\ 1 Cytogenetics and Molecular Genetics Laboratory, Istituto Auxologico Italiano, IRCCS, 20145 Milano, Italy; \\ guzzettisara@virgilio.it (S.G.); luciano.calza@gmail.com (L.C.); lucia.buccarello@marionegri.it (L.B.) \\ 2 Department of Agricultural and Environmental Sciences, Università degli Studi di Milano, 20133 Milano, \\ Italy; valentina.cesari@unimi.it (V.C.); ivan.toschi@unimi.it (I.T.) \\ 3 Laboratory of Clinical Neurobiology, Istituto Auxologico Italiano, IRCCS, 28824 Piancavallo-Verbania, Italy; \\ s.cattaldo@auxologico.it (S.C.); mauro@auxologico.it (A.M.) \\ 4 Division of Neurology and Neurorehabilitation, Istituto Auxologico Italiano, IRCCS, \\ 28824 Piancavallo-Verbania, Italy \\ 5 Department of Neurosciences, Università di Torino, 10126 Torino, Italy \\ 6 Experimental Laboratory of Immunological and Rheumatologic Researches, Istituto Auxologico Italiano, \\ IRCCS, 20145 Milano, Italy; francesca.pregnolato@gmail.com \\ 7 Department of Veterinary Medicine, Università degli Studi di Milano, 20133 Milano, Italy; \\ silvia.mazzola@unimi.it \\ * Correspondence: s.russo@auxologico.it; Tel.: +39-02-619113036; Fax: +39-02-619113033 \\ + These authors contributed equally to this work.
}

Received: 1 March 2018; Accepted: 30 March 2018; Published: 5 April 2018

\begin{abstract}
Angelman syndrome (AS, MIM 105830) is a rare neurodevelopmental disorder affecting 1:10-20,000 children. Patients show moderate to severe intellectual disability, ataxia and absence of speech. Studies on both post-mortem AS human brains and mouse models revealed dysfunctions in the extra synaptic gamma-aminobutyric acid (GABA) receptors implicated in the pathogenesis. Taurine is a free intracellular sulfur-containing amino acid, abundant in brain, considered an inhibiting neurotransmitter with neuroprotective properties. As taurine acts as an agonist of GABA-A receptors, we aimed at investigating whether it might ameliorate AS symptoms. Since mice weaning, we orally administered $1 \mathrm{~g} / \mathrm{kg} /$ day taurine in water to Ube3a-deficient mice. To test the improvement of motor and cognitive skills, Rotarod, Novel Object Recognition and Open Field tests were assayed at 7, 14, 21 and 30 weeks, while biochemical tests and amino acid dosages were carried out, respectively, by Western-blot and high-performance liquid chromatography (HPLC) on frozen whole brains. Treatment of $U b e 3 a^{m-/ \mathrm{p}^{+}}$mice with taurine significantly improved motor and learning skills and restored the levels of the post-synaptic PSD-95 and pERK1/2-ERK1/2 ratio to wild type values. No side effects of taurine were observed. Our study indicates taurine administration as a potential therapy to ameliorate motor deficits and learning difficulties in AS.
\end{abstract}

Keywords: Angelman syndrome; taurine oral administration; motor and learning recover of deficit

\section{Introduction}

Angelman syndrome (AS, MIM 105830) is a rare neurodevelopmental disorder affecting 1/10-20,000 children. The majority of AS patients share a moderate to severe development delay and intellectual disability, movement disorders and ataxia of gait, absence or limited speech and a peculiar behavior characterized by happy disposition, hyperexcitability and hand flapping, while over $80 \%$ of 
cases have relative microcephaly, seizures and a specifically altered EGG pattern with large-amplitude slow spike waves, sleep disorders [1,2]. Patients show a peculiar facies with prognathism, wide spaced teeth, pale skin and hair, addressing the clinical suspicion, but overall clinical manifestations are highly heterogeneous, partially depending on the specific molecular defects causing the disease. Figure 1 shows AS children at different ages and genetic defects evidencing the AS facies and its variability.

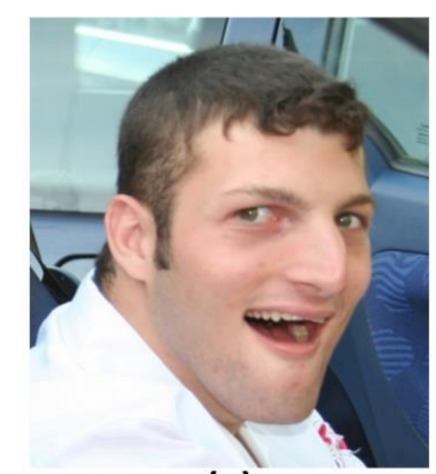

(a)

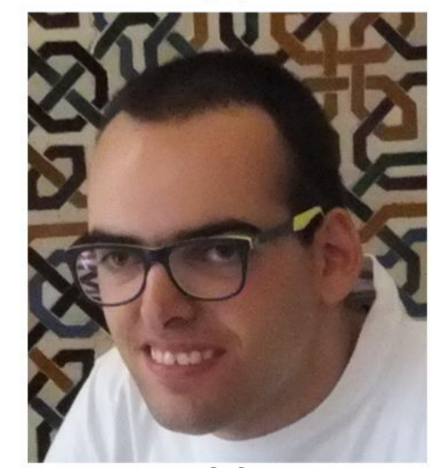

(c)

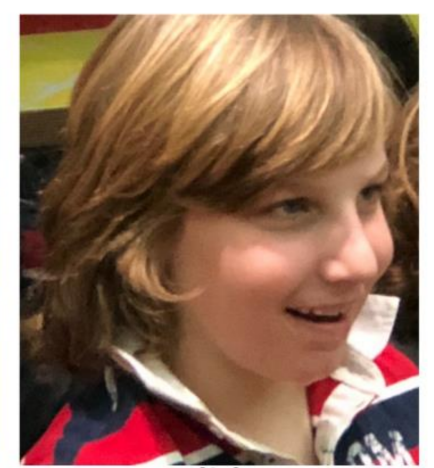

(b)

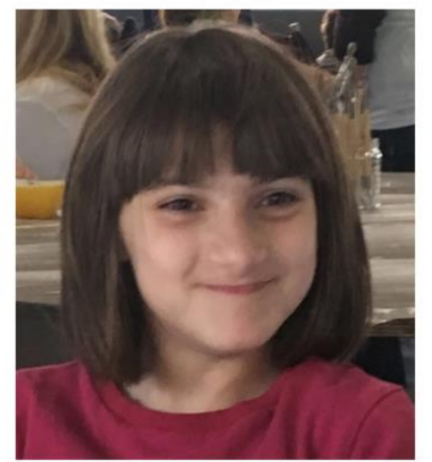

(d)

Figure 1. A photograph of AS patients representative of the genetic mechanisms: (a) deletion of 15q11-12; (b) chr.15 paternal uniparental disomy; (c) imprinting centre; (d) UBE3A point mutations. All patients' parents have signed the informed consent.

Genetic etiology consists of the lack of function of the maternal allele of UBE3A gene, mapping.within the imprinted chromosomal region 15q11.2-q13 and coding for the E3 ubiquitin protein ligase involved in the ubiquitination of targeted proteins via proteasome degradation. Expressed in multiple cell compartment, UBE3A regulates oxidative metabolism in mitochondria, gene transcription in euchromatin-rich nuclear domains and might be involved in synaptic plasticity [3]. A paternally neuron specific long antisense transcript prevents the transcription of the paternal UBE3A allele, so in neurons, in spinal cord and peripheral nerves only UBE3A maternal allele is expressed [4]. Four molecular classes of defects impair UBE3A functionality: deletions encompassing the gene on the maternal allele ( $70 \%$ of cases), paternal uniparental disomy of chromosome $15 q(3-5 \%)$, altered methylation at Imprinting Centre (3-5\%) and point mutations within UBE3A coding sequence in up to $11 \%$. Ube3a deficient mouse model [5] display phenotypes correlating with the human disorder, recapitulate features as motor deficits, learning and memory impairment, with differences depending on the strains. They greatly contributed to understand the molecular and cellular mechanisms involved in the pathophysiology of the disorder [6]. The potential dysfunction of GABAergic inhibitory system in Angelman syndrome has been investigated in animal model and in AS postmortem human brains revealing alterations that predict impairment of cortical extrasynaptic, but not synaptic GABAergic activity [7]. AS model mice also exhibit an increase in hippocampal mitochondrial reactive oxygen species which may contribute to the behavior deficit [8-10]. At the moment no efficacious therapy 
has been developed and the restoration of GABAergic neuronal function appears one of the most direct strategies to treat epileptic and neurological traits [11]. Administration of selective extrasynaptic GABA receptors agonist seems to ameliorate motor dysfunction in AS [12].

Taurine (2- $\beta$ aminoethansulphonic acid) is a free intracellular sulfur-containing amino acid characterized by the presence of a sulfonate instead of a carboxyl group, not incorporated in proteins and representing the second most abundant amino acid in Central Nervous System (CNS) [13]. In human, taurine biosynthesis occurs primarily in the liver, but it may be consumed through food, mainly meat and seafood [14]. In CNS it exerts roles in cell volume regulation and in neurotransmission. Recently, various studies evidenced that taurine plays a neuroprotective role for CNS [15-17] and it is involved in neurogenesis [18]. Essential for the neuroprotection is its role in opening inhibitory anionic channels by activating glycine and/or non-synaptic GABA-A receptors [19]. Reyes-Haro et al. (2014) [20] showed taurine acts as weak agonist of GABA-A receptors in astrocytes and in STC-1 (Secretin tumor cell lines), cells expressing various GABA-A subunits.

On the basis of this evidence we designed the present study to investigate the efficacy of taurine administration to $U b e 3 a^{m-/ \mathrm{p}+}$ mice in ameliorating their motor and learning dysfunction. Basing on reports showing that taurine, chronically administered both in food [21] or in drinking water $[15,22,23]$ ameliorated motor and/or cognitive deficits of syndromic disorder, we orally administered $1 \mathrm{~g} / \mathrm{kg} /$ day weight. A similar dosage has already been demonstrated successful and not toxic in a mouse model of Alzheimer's disease [15]. Considering that the rescue of AS neurocognitive deficits achieved best results in an AS inducible mouse, when UBE3A was reactivated at three weeks [24], we chose to start taurine administration at the 21 st day, just after weaning.

\section{Results}

Therapeutic effect of oral and chronic administration of taurine in motor impairment and learning difficulties of Angelman syndrome was examined administrating a battery of three behavioral tests to the transgenic Ube3a $a^{m-/ p^{+}}$on the C57BL/ 6 background. Eight different experimental groups of animals were considered: untreated (1) male and (2) female Ube3a $a^{m-/ p+}$; treated (3) male and (4) female Ube $3 a^{m-/ \mathrm{p}^{+}}$; untreated (5) male and (6) female wild type Ube3a $a^{m+/ \mathrm{p}+}(\mathrm{wt})$ and treated (7) male and (8) female $U b e 3 a^{m+/ p^{+}}$wt.

\subsection{Taurine Ameliorates Motor Coordination of Ube $3 a^{m-/ p+}$ Mice}

To identify differences in motor coordination, all the test groups were assayed through rotarod tests at four development time points: 7, 14, 21 and 30 weeks after birth. As showed in Figure 2, comparison between groups, untreated $U b e 3 a^{m-1 p+}$ versus untreated wt mice, showed a significant impairment in rotarod performance (all time points: ${ }^{* *} p<0.001$ ), while $U b e 3 a^{m-/ \mathrm{p}^{+}}$mice administered with taurine since weaning showed a heterogeneous but significant amelioration. The only exception was observed for Ube3a $a^{m-/ p^{+}}$male group at 30 weeks, showing no significant improvement. No significant effect was observed in controls after taurine treatment.

\subsection{Open Field Test Reveals a Partial Improvement upon Taurine Administration}

The open field test (OFT) was used to evaluate locomotor activity, anxiety and exploration behavior in our groups: total distance, distance travelled in the center zone and number of rearings were then taken into account. Analysis of these parameters obtained by the ANY Maze software, indicated a behavioral deficit in the untreated Ube $3 a^{m-1 p+}$ versus untreated wt mice as revealed by the significant reduction of all observed OFT parameters (Figure 3). Since the 14th week, a significant decrease in total distance was observed (in both males and females) (Figure 3a-left and right panels), but it was more evident in females. Similarly, the significant differences in distance traveled in the central area as well as rearing number were more pronounced in females and became evident since the 7th week of life (Figure 3b,c-right panels). 


\section{Rotarod}
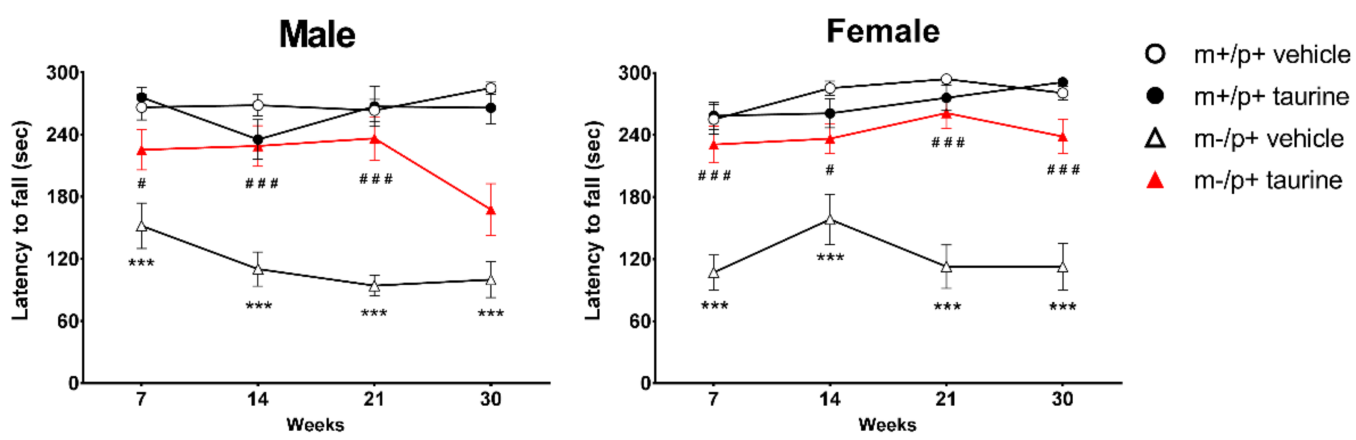

Figure 2. Taurine treatment improved motor coordination in AS $\left(U b e 3 a^{m-/ p+}\right)$ mice. Rotarod tests were carried out at four time points $\left(7,14,21\right.$ and 30 weeks) on wild type (wt) and Ube3a $a^{m-1 p+}$ mice (both treated (taurine) and untreated (vehicle)). Analyses were conducted by separating genders (male: left panel; female: right panel). Animals per group: male mice $(\mathrm{wt}+$ vehicle $=14, \mathrm{wt}+$ taurine $=12$, Ube $3 a^{m-/ p+}+$ vehicle $=11, U b e 3 a^{m-/ p+}+$ taurine $\left.=11\right) ;$ female mice $(\mathrm{wt}+$ vehicle $=15, \mathrm{wt}+$ taurine $=13$, Ube $3 a^{m-/ p+}+$ vehicle $=12$, Ube3a $a^{m-/ p+}+$ taurine $=14$ ). Data are shown, for each time point, as media \pm SEM. Effect of genotype: ${ }^{* *} p<0.001$; effect of taurine treatment on Ube $3 a^{m-/ p+}$ genotype: \# $p<0.05,{ }^{\# \#} p<0.001$.

Taurine treatment showed a beneficial effect mainly in female Ube3a $a^{m-1 p+}$ mice: a significant increase in locomotor activity was observed at the 14th and 21th week (\#\#\# $p<0.001$ ) (Figure 3a—right panel). Taurine-treated Ube $3 a^{m-/ p+}$ males showed a partial but not significant rescue of locomotor performances (Figure 3a-left panel). Similarly, differences between genotypes in central area distances (Figure $3 \mathrm{~b}$-right panel) and rearing number (Figure $3 \mathrm{c}$-right panel) were partially restored by taurine at the 14th and 21th week in Ube3a $a^{m-/ p+}$ female group ( ${ }^{\#} p<0.05$; $\left.{ }^{\# \#} p<0.01\right)$. A partial restore was also noted in males group, at the 7th week for central square distance (Figure $3 \mathrm{~b}$-left panel) and at the 30th week for rearing number (Figure $3 c$ - left panel). For all parameters, no significant effect of taurine was observed in wt animals.

From a longitudinal view, a general flexion in measured parameters was observed in almost all test groups from the first time point (7th week) to other time points (14th and/or 21th and/or 30th week).

\subsection{Learning and Memory Deficits Improve in Ube3a ${ }^{m-1 p+}$ after Taurine Administration}

Novel object recognition test (NORT) was used to measure visual recognition memory and anxiety level in Ube3a $a^{m-1 p+}$ mice along with $U b e 3 a^{m+/ p+}$ controls. Mice show an innate and natural ability to explore unfamiliar objects: low levels of exploration may correlate with a state of anxiety. Locomotor activity should be carefully monitored but is not a critical element that can invalidate the effectiveness of the NORT [25]. Hyperdinamic and non-interacting mice have been discarded or retested. Evaluation of recognition index (RI) (Figure 4) revealed a strong genotype effect (Ube3a $a^{m-/ p+}$ vs. wild type) evidencing, for both males and females, at each time point, a very limited skill to explore the unfamiliar object $\left.{ }^{* * *} p<0.001\right)$. Ube3a ${ }^{m-/ p+}$ mice administered with taurine ameliorated their propensity for exploration: Ube3a $a^{m-/ p+}$ male mice at the 21th and 30th weeks (\#\#\# $p<0.001$ ); Ube3a $a^{m-1 p+}$ female mice at the 7th (\# $p<0.05), 21$ th (\#\#\# $p<0.001$ ) and 30th (\# $p<0.01)$ weeks. Surprisingly, at the 30th week Ube $3 a^{m+/ p+}$ female mice manifested a slight and borderline decrease in novel object preference. Longitudinal analyses highlighted, for both genders, the maximum improvement in the 30th week.

\subsection{Effect of Taurine on Synaptic Markers}

To investigate if taurine administration may restore the synaptic deregulations pointed out by studies on AS patients [26-28], the level of the PSD-95, and the ratio between pERK1/2 and total ERK1/2 were investigated by Western blot in total brains from 30-week-old animals. As shown in 
Figure 5a,b, a slight increase of total PSD-95 (black bar) and activation of the ERK1/2 ratio (black bar) was found in transgenic mice. Taurine administration to transgenic mice restored values to wild type levels (red bar). Consequently, another member of MAPK signaling, transducing extracellular stimuli in intracellular cascade, JNK (c-Jun N-terminal kinase) was assayed showing a normal pJNK/JNK ratio in Ube $3 a^{m-/ p+}$ whole brain.

\section{Open field}

\section{a)}
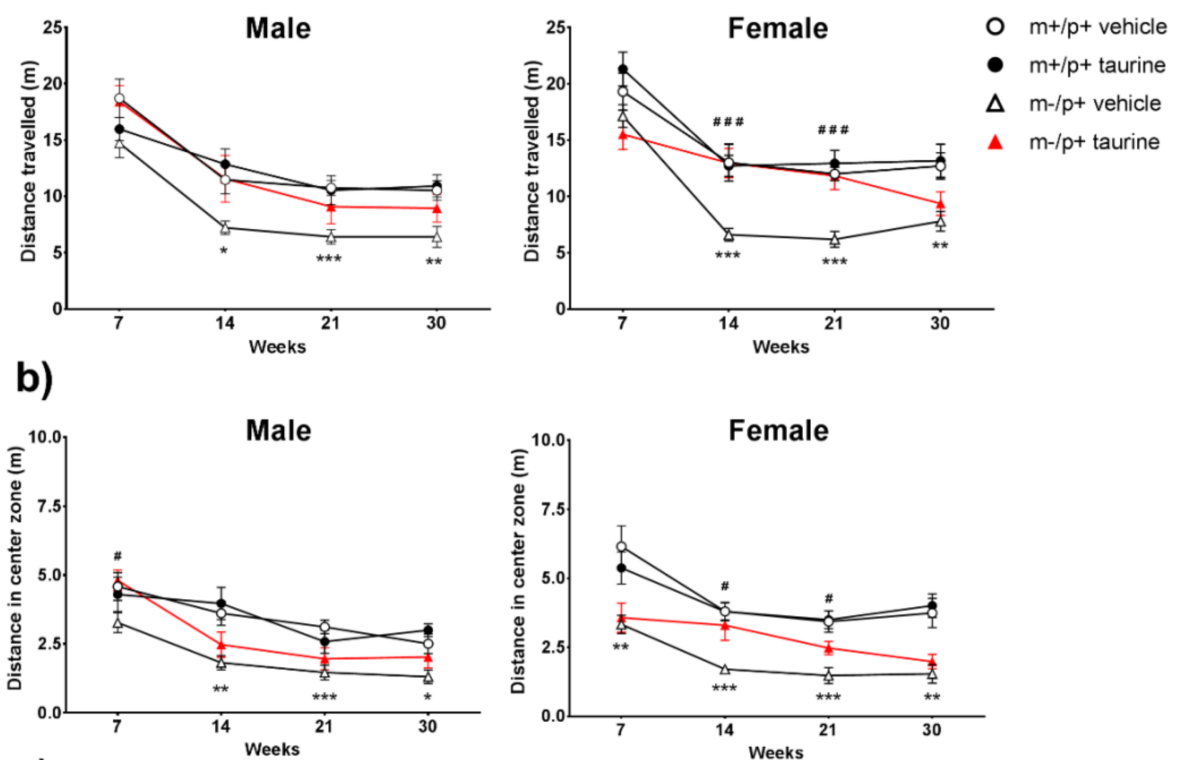

c)
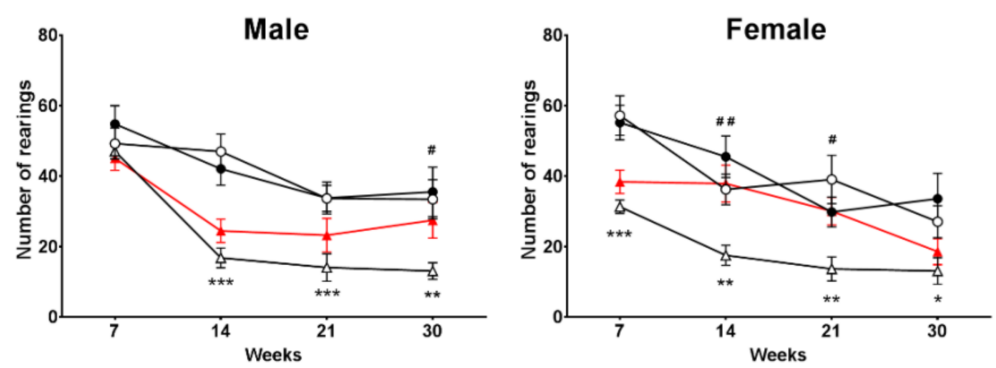

Figure 3. Taurine administration partially improved locomotor activity, anxiety and exploration in AS female $\left(U b e 3 a^{m-/ p+}\right)$ mice. Open field assay was carried out at four time points $(7,14,21$ and 30 weeks) on wild type (WT) and Ube3 $a^{m-1 p+}$ mice (both treated (taurine) and untreated (vehicle)) by measuring (a) total distance traveled (m); (b) distance in center square (m) and (c) number of rearings. Analyses were conducted by separating genders (male: left panels; female: right panels). Number of animals per group: male mice $\left(\mathrm{wt}+\right.$ vehicle $=13, \mathrm{wt}+$ taurine $=12, U$ be $3 a^{m-/ p+}+$ vehicle $=11, U b e 3 a^{m-/ p+}+$ taurine $=12$ ); female mice $\left(\mathrm{wt}+\right.$ vehicle $=13, \mathrm{wt}+$ taurine $=13, U$ be $3 a^{m-/ p+}+$ vehicle $=11$, Ube $3 a^{m-/ p+}+$ taurine =13). Data are shown, for each time point, as media \pm SEM. Effect of genotype: ${ }^{*} p<0.05,{ }^{* *} p<0.01$, *** $p<0.001$; effect of taurine treatment on Ube $3 a^{m-1 p+}$ genotype: ${ }^{\#} p<0.05,{ }^{\# \#} p<0.01,{ }^{\# \#} p<0.001$.

\subsection{Taurine Content in Brain and Serum}

We examined if the daily consumption of taurine in water altered the taurine content in serum and in whole brain. As shown in Figure 6, there were no differences in taurine level between untreated transgenic and wild type mice. A significant increase was observed in serum, where treated mice, independently from genotype, showed a taurine concentration 4-6-fold higher (Figure 6a). Conversely, the measurements of taurine in brain were similar in all tested groups (Figure 6b), according to the 
regulation of brain concentration by an active TauT transporter [16,29]. Quantification of additional neurotransmitters as Glutamic acid, GABA and Glutamine did not show significant differences (Appendix A, Figure A1).

\section{Novel-object recognition}
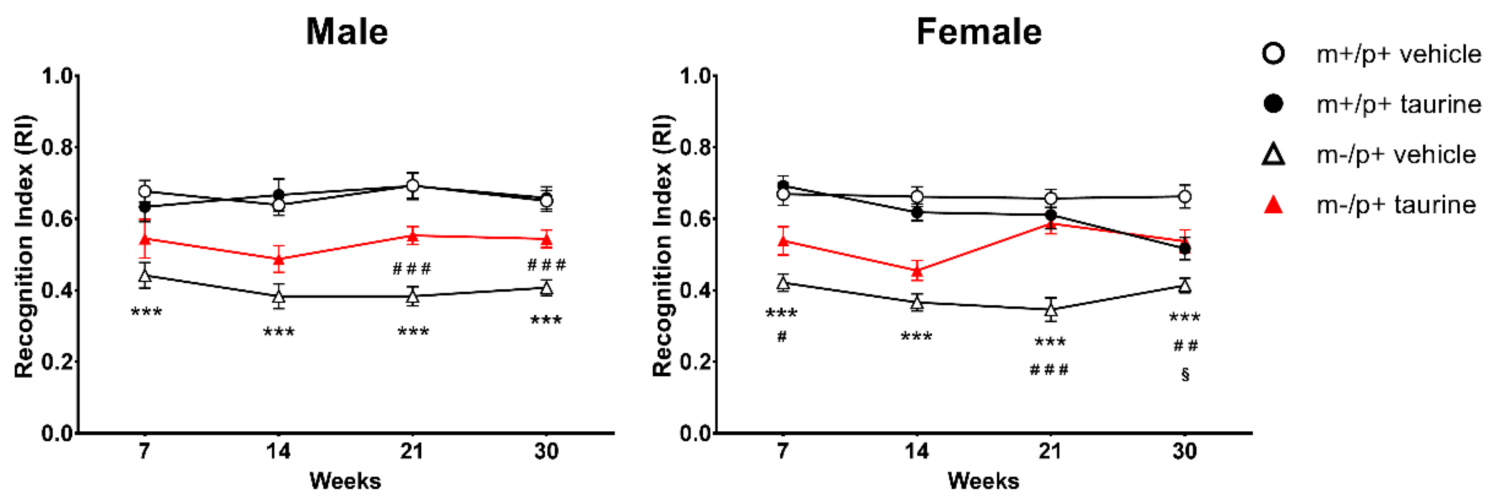

Figure 4. Taurine treatment improved learning and memory in AS (Ube3a $\left.a^{m-1 p+}\right)$ mice. Novel object recognition test was carried out at four time points $(7,14,21$ and 30 weeks) on wild type (WT) and Ube $3 a^{m-/ p+}$ mice (both treated (taurine) and untreated (vehicle)). Analyses were conducted by separating genders (male: left panel; female: right panel). Number of animals per group: male mice $\left(\mathrm{wt}+\right.$ vehicle $=14, \mathrm{wt}+$ taurine $=11, U b e 3 a^{m-1 p+}+$ vehicle $=14, U b e 3 a^{m-/ p+}+$ taurine $\left.=13\right)$; female mice $\left(\mathrm{wt}+\right.$ vehicle $=12, \mathrm{wt}+$ taurine $=14$, Ube $3 a^{m-/ p+}+$ vehicle $=12, U b e 3 a^{m-/ p+}+$ taurine $\left.=14\right)$. Data are shown, for each time point, as media \pm SEM. Effect of genotype: ${ }^{* *} p<0.001$; effect of taurine treatment on Ube3a $a^{m+/ p+}$ genotype: ${ }^{\S} p<0.05$; effect of taurine treatment on Ube3a $a^{m-p+}$ genotype: \# $p<0.05,{ }^{\# \#} p<0.01,{ }^{\# \# \#} p<0.001$.

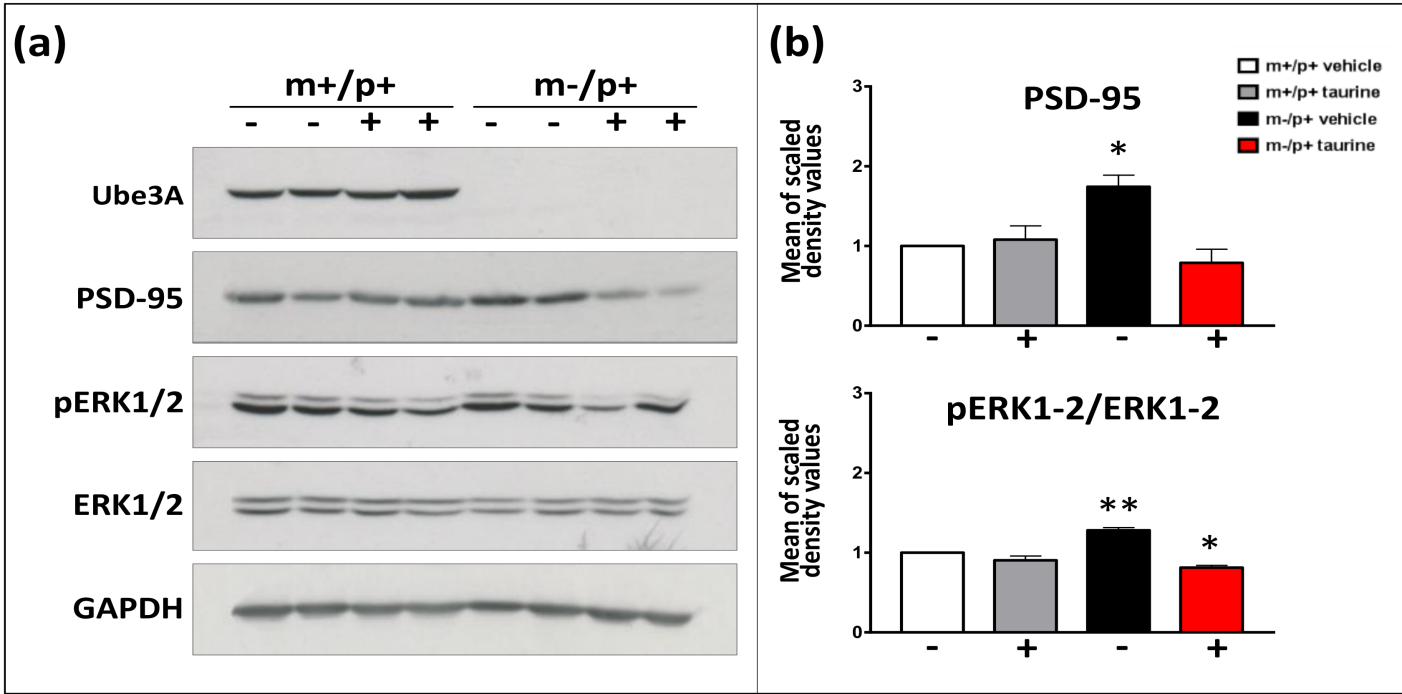

Figure 5. Activation of pERK1/2-ERK1/2 signaling and altered level of PSD-95 restored by taurine administration. Panel (a) Representative Western blots from female total brain homogenates show the expression of UBE3A (top blot), PSD-95, phosphorylated ERK1/2 and total ERK1/2 proteins, normalized by GADPH. $\mathrm{m}-/ \mathrm{p}+\left(\right.$ for $\left.U b e 3 a^{m-/ p+}\right)$ and $\mathrm{m}+/ \mathrm{p}+\left(\right.$ for $\left.U b e 3 a^{m+/ p+}\right)$ indicate the respective genotypes; the signs just below are referred to the treatment: + for taurine administration and-for vehicle. Panel (b) mean of scaled density values obtained from 3 independent experiments are showed. Significant differences between groups and untreated control are displayed with asterisks $\left({ }^{*} p<0.05\right.$, ** $p<0.01)$. 


\section{Taurine dosage (HPLC)}

a) Blood serum

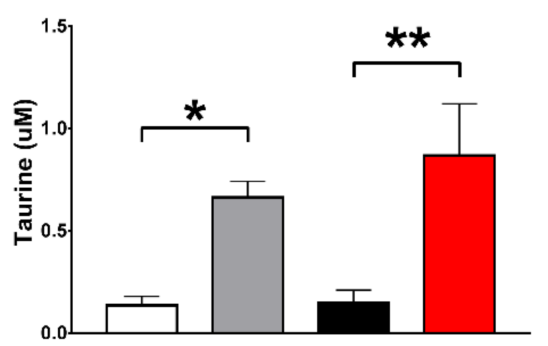

b) Brain $\square+/ p+$ vehicle

$\square \mathrm{m}+\mathrm{p}+$ taurine

$m-/ p+$ vehicle

$\mathrm{m}-\mathrm{p}+$ taurine

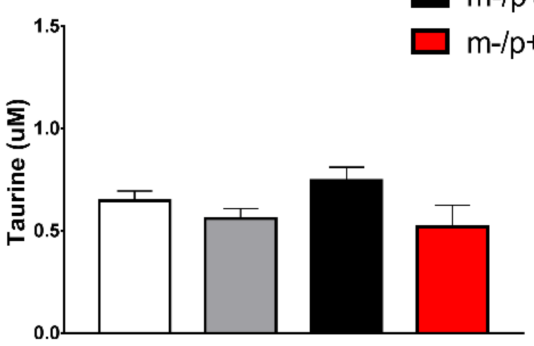

Figure 6. Blood serum Panel (a) and whole brain Panel (b) content of taurine evidenced a different accumulation in the two tissues. Measurement of taurine levels in blood serum and in whole brain of mice evaluated by high performance liquid chromatography. Asterisks indicate significant differences $\left({ }^{*} p<0.05,{ }^{* *} p<0.01\right)$. Number of mouse blood serums: $\mathrm{wt}+$ vehicle $=8, \mathrm{wt}+$ taurine $=10$, Ube $3 a^{m-/ p+}+$ vehicle $=6, U b e 3 a^{m-/ p+}+$ taurine $=6$. Number of mouse brains: $\mathrm{wt}+$ vehicle $=13$, $\mathrm{wt}+$ taurine $=8, U b e 3 a^{m-/ p+}+$ vehicle $=7, U b e 3 a^{m-/ p+}+$ taurine $=9$.

\section{Discussion}

Angelman syndrome is a rare disorder severely affecting AS probands' and their parents' quality of life. Cognitive impairment, ataxic gait, absence of speech and occurrence of seizures are the main features of the syndrome that no therapy is available to ameliorate.

Our study reports that taurine dissolved in drinking water may restore motor deficits and learning impairment of a Ube3a $a^{m-1 p+}$ C57BL/6J mouse when administered daily, while the same treatment has no effect on motor and cognitive behavior of a wild type Ube3a $a^{m+p+}$ mouse. As the best period to obtain a rescue of behavioral deficits in the AS model seems to be the postnatal period [24], we started the treatment in the juvenile period of life (three weeks) as soon as the pups were able to feed by themselves. During the statistical evaluation, genders were considered separately to ensure a better homogeneity in the test groups and the amelioration appeared substantially overlapping, with the exception of the Open Field test. The rescue of motor deficit demonstrated by rotarod assay appeared since the first set of tests at the 7th week suggesting a strong effect of taurine on these skills; this point is supported by the performances at total distance walked in the OF test, a measure of ataxia attenuation, with significant improvement in the female group and a trend to improvement in males. A slight decrease of female performance at the last time-point may suggest that dosage could need to be adjusted during such a long chronic treatment. Variations among sex might be due to some environmental factors, as reported by Kovacs [30] in C57BL/6J mice. Memory and learning skills, as shown by NORT, results raised toward those of control animals, since the last two sets of evaluations, indicating the need of a longer treatment to achieve visible improvement of learning disability. Apparently, the prolonged administration of $1 \mathrm{~g} / \mathrm{Kg} /$ day taurine did not reveal any toxicity [15]. Taurine is well known to increase neurogenesis of adult neural stem cells [18,31] and to promote neuroprotection preventing (i) glutamate-induced excitotoxicity through modulation of intracellular calcium homeostasis [32]; (ii) mitochondrial dysfunction by exerting an antioxidant activity; and (iii) apoptosis, by downregulating molecules that drive apoptotic events $[16,17,33,34]$. Potential beneficial effects of taurine in brain disorders, as Alzheimer's [15], schizophrenia [16] and neurodevelopment disorder as fragile $X$ syndrome have been reported [23,35]. Out of the multiple functions of taurine in the mammalian body, a key one is modulation of GABA neurotransmission. A defect in tonic GABAergic inhibition shared by fragile $X$ and Angelman syndrome has been suggested to contribute to expression of the disease phenotype [11], raising the option to use agonists of GABA receptors as a winning strategy to restore the decrease in GABAergic inhibition [12]. Interestingly, 
taurine is considered an endogenous ligand of GABA extrasynaptic receptors [36]. Our study first investigates and proves the beneficial effect of taurine administration in ameliorating AS features of Ube3a $a^{m-1 p+}$ mice supporting the concept that GABA receptor agonist strategy may be effective. Neurological deficits in Angelman syndrome have been associated with an increased level of Arc (activity-regulated cytoskeletal-associated protein) which has the consequence to impair the Long Term Potentiation (LTP) and alter the recruitment of the scaffold protein PSD-95 (PostSynaptic Density-95), a marker of synaptic dysfunction [26]. Furthermore, UBE3A loss enhances the ERK1/2 (Extracellular Signal-Regulated Kinase) signaling pathway [27], which plays a relevant role in several biological processes including synaptic activity with a stimulatory effect on the proliferation of neural stem cells rather than in the following differentiation [37]. Our biochemical analyses confirmed the activation of ERK1/2 pathway and a major expression of the synaptic protein PSD-95 as a consequence of UBE3A deficit in the Ube3a $a^{m-/ p+}$ mice and demonstrated that taurine may restore a normal activity of both markers. We could speculate that the therapeutic effect of taurine in the AS model might be achieved for a restoration of both extrasynaptic GABAergic activity, with taurine being an agonist of the GABA receptor, and synaptic pathway as shown by our data on ERK1/2 activity and PSD-95. The dosage of taurine in the whole brain remained the same in treated and untreated animals while in serum we observed an a 4-6 folds increase. This finding is not completely unexpected considering the occurrence of active transport of taurine in brain, mediated by a specific saturable TauT transporter [16]. Sved D.W. et al. [29] investigated the absorption and tissue distribution when taurine was orally administered to rats using ${ }^{14} \mathrm{C}$ taurine and observed that some tissues as brain, heart, muscles showed a slow increase of ${ }^{14} \mathrm{C}$ taurine during the first $24 \mathrm{~h}$ and a small decrease during the following $168 \mathrm{~h}$ without changing the total amount of brain taurine. The authors supposed that after taurine passed the blood-brain barrier (BBB), it became part of an endogenous pool regulated by a $\mathrm{Na}^{+} / \mathrm{Cl}^{-}$saturable transport within brain cells, which makes the taurine concentration in brain not proportional to plasma concentration. The same behavior has been described in muscles where despite a very high dosage of taurine being administered to a Duchenne dystrophy model, high performance liquid chromatography (HPLC) quantification revealed a higher concentration only in serum and in liver [38], while muscles maintained a constant concentration.

\section{Materials and Methods}

\subsection{Animals}

All subjects were heterozygous mice with deficiency of Ube3a on the maternal allele (Ube3a $a^{m-1 p+}$ ) and wild type $\left(U b e 3 a^{m+/ p+}\right)$ littermates, on the C57BL/6J background. Mice, developed by the Beaudet laboratory [39], were acquired from Charles River laboratories. Heterozygous $U b e 3 a^{m-1 p+}$ females were

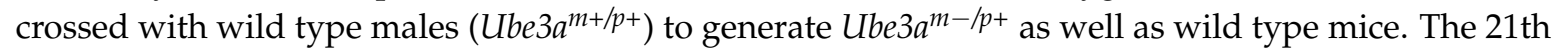
day after birth, the animals were weaned and genotyped by PCR (from tail tissues), as described in [39]. Animals were bred with a regular 12:12 h light/dark cycle (lights on 07:00 a.m.), at a constant room temperature of $22 \pm 2{ }^{\circ} \mathrm{C}$, and relative humidity approximately $55 \pm 10 \%$. Animals were housed $(n=4$ per group) in standard mouse cages. All mice were provisioned with bedding material (hard wood shavings), ad libitum food (Global Diet 2018S, Harlan Italy) and water. All procedures involving animals and their care were conducted in strict compliance with the national and international laws and policies and approved by the University of Study of Milano Animal Care and Use Committee (IACUC) (Approval Number: 95/14; Approval Date: 27 March 2014).

\subsection{Tissue Collection}

After behavioral experiments, mice at the 7th and 30th weeks were euthanized by cervical dislocation and the brains were immediately frozen in dry ice and stored at $-80{ }^{\circ} \mathrm{C}$ prior to biochemical and immunohistochemical processing. 


\subsection{Taurine Administration}

Taurine was orally administered via drinking water at dose level of $1000 \mathrm{mg} / \mathrm{kg} /$ day from weaning to 30 weeks of age. Taurine-based solution was prepared fresh twice a week adjusting volumes and amount of dissolved taurine for body weight changes and water intake Body weights were monitored twice a week for all the treated groups. To ensure the mice received the right dose required by the protocol, the effective amount of taurine intake was inspected every day for each cage. The animals did not suspend the taurine administration before tests, apart from the time they were trained or tested.

\subsection{Test Groups}

Four groups were considered for tests. Group 1: Ube3a $a^{m+/ p+}$ received drinking water only (control);

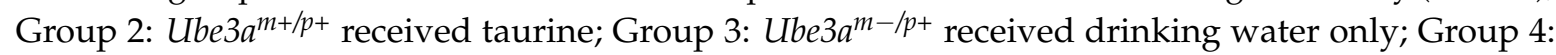
$U b e 3 a^{m-/ p+}$ received taurine. Males and females were statistically analyzed separately.

\subsection{Behavioral Tests}

Tests were performed at 7th, 14th, 21th and 30th week of life. The equipment (e.g., open field arena) and all objects directly interacting with animals were cleaned using $70 \%$ ethyl alcohol to eliminate distracting/confounding olfactory stimuli.

\subsubsection{Rotarod}

Mice were tested for balance and motor coordination by using an accelerating Rotarod (Ugo Basile 7650 model). Animals were initially trained for 4 sessions at a constant speed of $4 \mathrm{rpm}$ : when properly trained they were tested twice (a 5 min-test) on a gradually accelerating rotarod $(0.3 \mathrm{rpm} / \mathrm{s}$ increase from 3 to $300 \mathrm{rpm}$ ) by measuring the latency to fall from the bar (seconds). The final score was determined as the average of 2 trials.

\subsubsection{Open Field (OF) and Spontaneous Locomotor Activity}

The open field test evaluates general locomotion activity, exploration behavior and level of anxiety by exposing mice to a novel and open space [40]. We used a $40 \mathrm{~cm} \times 40 \mathrm{~cm} \times 40 \mathrm{~cm}$ grey arena divided into 25 equal squares by black lines. During test, noises and lighting were consistently attenuated in order not to create artificial/external stress to mice. Mice were placed into the center of the floor defined as a "starting point" and allowed to explore for $5 \mathrm{~min}$. Tests were video-recorded by a camera apparatus. Test parameters were obtained via the automated ANY-maze Behavior Tracking Software (Stoelting Co., Wood Dale, IL, USA). Total distance, distance in central square and number of rearings were taken into account.

\subsubsection{Novel-Object Recognition Test (NORT)}

The novel-object recognition test (NORT) is a memory test relied on spontaneous animal behavior [41,42] aimed to exploit the natural exploration attitudes of mice. In the present study, tests were conducted in an open-field arena $(40 \mathrm{~cm} \times 40 \mathrm{~cm} \times 40 \mathrm{~cm})$ divided into 25 equal squares by black lines. The first day (day 1), animals were habituated for five minutes in a clean and empty arena: their movements were recorded as the number of line crossings, which provided an indication of both wild type and transgenic mice locomotion motor activities. In the next day (day 2), mice were re-placed in the same arena containing two identical objects (familiarization phase), randomly selected from a group of specific objects (a red rubber cylinder $(4 \mathrm{~cm} \times 5 \mathrm{~cm}$ ), a plastic vial with a white cup $(3 \mathrm{~cm} \times 6 \mathrm{~cm})$ and a plastic cube $(3 \mathrm{~cm} \times 5 \mathrm{~cm}))$ to avoid bias among animals and between groups. Exploration was recorded in a $10 \mathrm{~min}$ trial by an investigator blinded to the genotype and treatment. In the familiarization phase, the time spent exploring the two identical objects (e.g., sniffing, touching, and stretching - at a minimum distance of $2 \mathrm{~cm}$ ) was recorded and evaluated as object investigation. The test day (day 3), mice were still placed in the arena and exposed for $5 \mathrm{~min}$ to two different 
objects: one exposed during the familiarization phase, while the second one was something absolutely unfamiliar. As described above, time spent interacting with both objects was recorded and evaluated. A cut-off of $6 \mathrm{~s}$ was applied to the total exploration time (both during the familiarization and test phases): tests not meeting the minimum threshold were discarded. Recognition Index (RI), expressed as time spent exploring the novel object (TN) with respect to the total time spent exploring both objects $(\mathrm{TN}+\mathrm{TF})$ was estimated.

\subsection{Western Blotting}

Approximately $200 \mathrm{mg}$ of brain tissue, were rapidly homogenized in ice-cold lysis buffer (Sucrose 0.32 M, Hepes $10 \mathrm{mM}$ pH 7.4, PMSF $0.1 \mathrm{mM}, \mathrm{NaHCO}_{3} 1 \mathrm{mM}$, in presence of proteinase and phosphatase inhibitors, Complete Mini and PhosSTOP-Roche, Basel, Switzerland) using a mechanical homogenizer (Euro Turrax T20b, Ika Labor Technik, Staufen im Breisgau, Germany) at 27,000 rpm for $1 \mathrm{~min}$, holding the sample on ice. Total homogenates were clarified by centrifugation at $1000 \times g$ for 5 min at $4{ }^{\circ} \mathrm{C}$ to remove crude nuclear material (P1). The supernatant (S1) was assayed for total protein content using a commercial protein assay kit according to manufacturer's protocol (DC Protein Assay Kit-BioRad, Hercules, CA, USA).

Samples containing $30 \mu \mathrm{g}$ proteins were resolved in $8 \%$ sodium dodecyl sulphate-polyacrylamide gels under reducing conditions and transferred to nitrocellulose membranes (iBlot Gel Transfer Stacks Nitrocellulose-Invitrogen, Carlsbad, CA, USA). Membranes were blocked for $1 \mathrm{~h}$ at room temperature in $5 \%$ milk solution, TBS (Tris-NaCl, $\mathrm{pH} 7.5$ ) and tween $0.1 \%$ (TBT-T) and, then incubated with primary antibodies UBE3A (1:500; polyclonal; Proteintech Group, Rosemont, IL, USA), PSD-95 (1:1000; monoclonal; Millipore, Burlington, MA, USA), p44/42 MAPK (Erk1/2) (1:2000; polyclonal; Cell Signaling Technology, Danvers, MA, USA), Phospho-p44/42 MAPK (Erk1/2) (Thr202/Tyr204) (1:2000; monoclonal; Cell Signaling Technology), SAPK/JNK (1:1000; polyclonal; Cell Signaling Technology), Phospho-SAPK/JNK (1:1000; polyclonal; Cell Signaling Technology) and GAPDH (1:1500; polyclonal; Santa Cruz Biotechnology, Dallas, TX, USA) at $4{ }^{\circ} \mathrm{C}$ overnight in $5 \%$ milk TBS-T solution. Subsequently, membranes were washed and incubated for $1 \mathrm{~h}$ at room temperature in $5 \%$ milk, TBS-T solution with peroxidase-conjugated anti-rabbit or anti-mouse immunoglobulin G (Millipore) as secondary reagents. After washing with TBS-T, membranes were treated with ECL (Novex ECL Chemiluminescent Substrate Reagent Kit, Invitrogen). X-ray films were exposed in a linear range. Bands were quantified by using ImageJ software 1.8.0 (Available online: rsb.info.nih.gov/ij). Density value of each band was normalized against housekeeper protein GAPDH. Fold changes (with respect to control group) were calculated as the ratio of the average of normalized densities of each group $(\mathrm{n}$ per group $=2)$ and the average of the controls. Experiments were performed in triplicate.

\subsection{HPLC Method}

\subsubsection{Chemicals and Reagents}

All chemicals and solvents were of analytical-reagent grade and used without further purification. Methanol HPLC grade was from VWR International, sodium acetate and 2-O-phthaldialdehyde was from Sigma-Aldrich (St. Louis, MO, USA).

\subsubsection{Samples Preparation}

Brains were stored at $-80^{\circ} \mathrm{C}$ until analysis. Tissues were homogenized in 5 volumes $(w / v)$ of methanol: $0.1 \mathrm{M}$ Perchloric Acid 1:1 and then centrifuged for $5 \mathrm{~min}$ at $3000 \times \mathrm{g}$ at $4{ }^{\circ} \mathrm{C} .40 \mathrm{~mL}$ of supernatants were then used for derivatization procedure.

\subsubsection{Derivatization Procedure}

The standards or samples were precolumn derivatized with 2-O-phthaldialdehyde (OPA) reagent solution. The derivatization reagent was freshly prepared every week and protected from light 
exposure, as described by Perucho in a previous study [43]. After derivatization, $35 \mu \mathrm{L}$ of the mixture was injected into the analytical system.

\subsubsection{Chromatography System}

An Agilent 1100 series HPLC system (Santa Clara, CA, USA) equipped with a Fluorescence Detector (flow-cell volume of $8 \mu \mathrm{L}$ ) was used for this study. The detector excitation and emission wavelengths were set at 240 and $450 \mathrm{~nm}$, respectively. The Synergi Hydro-RP analytical column $(80 \AA, 150 \mathrm{~mm} \times 2 \mathrm{~mm} \times 4 \mu \mathrm{m})$ was used as stationary phase at ambient temperature [44]. Gradients were prepared with two degassed solvent mixtures. Solvent A was $0.05 \mathrm{M}$ sodium acetate buffer, $\mathrm{pH} 5.88$, and methanol (95:5), and Solvent B was methanol and double-distilled water (70:30). Gradient program was set as follows: initial conditions $25 \% \mathrm{~B}$, linear step to $25 \%$ B from 0.1 to $3 \mathrm{~min}$, gradient step to $33 \% \mathrm{~B}$ in $6.5 \mathrm{~min}$, gradient step at $60 \% \mathrm{~B}$ for $2.5 \mathrm{~min}$ duration, jump to $25 \% \mathrm{~B}$ in $0.1 \mathrm{~min}$; flow rate of $0.5 \mathrm{~mL} / \mathrm{min}$. Taurine concentration was calculated by comparison with calibrated external standard solution $(1 \mu \mathrm{M})$.

\subsection{Statistical Analyses}

Statistical analyses were performed by using computing environment $\mathrm{R}$ and related specific "packages". Graphics (plots and histograms) were generated by using GraphPad Prism (V.7) software. Values are presented as mean \pm SEM. For all statistical approaches, a $p$-value $<0.05$ was considered significant.

\subsubsection{Behavioral Tests}

Data were analyzed by fitting General Estimating Equations (GEE) [45] using "geeglm" function (library "geepack") with an AutoRegressive Order 1 (AR1) correlation structure and link = "identity". Wald $\chi^{2}$ tests were conducted to evaluate the effect of genotype, treatment and time point (week) and their interactions. For each time point, individual group means were then compared using Tukey's method to adjust $p$-values. For all behavioral tests, only mice with measurements in at least three time points were included in the analyses.

\subsubsection{Neurotransmitters Dosage}

To evaluate differences in neurotransmitters (Taurine, Glutamic acid, Glutamine and GABA) levels in mouse brain and serum (only for taurine), inter-group comparisons were carried out by using two-way ANOVA, followed by TukeyHSD post hoc test.

\subsubsection{Western-Blot}

Fold change differences with respect to untreated wild type group were analyzed by using a one-way ANOVA followed by TukeyHSD post hoc test.

\section{Conclusions}

This study first explores and proves the therapeutic effect of the sulfur-containing amino acid taurine, neurotransmitter agonist of GABAergic transmission involved in neurogenesis and neuroprotection, able to rescue the motor and learning deficit in a mouse model of the rare neurodevelopment disorder Angelman syndrome. The investigation of the involved mechanisms pointed to a restoration of the activation of the ERK pathway and of extrasynaptic GABAergic inhibition. The well-known role of taurine in preventing mitochondrial oxidative stress and inhibiting apoptosis as well as the proved enhancement of adult neural stem cells might also contribute to ameliorate the behavior deficits of AS model. 
Acknowledgments: We would like to thank families of Angelman patients for their support. The study has been supported by OR.S.A. (Organizzazione Sindrome di Angelman) Grant to Istituto Auxologico Italiano and by MOH Grants to Istituto Auxologico Italiano (08C002).

Author Contributions: Silvia Russo and Silvia Michela Mazzola conceived the project and designed the experiments; Sara Guzzetti performed genotyping and Western-blot experiments; Lucia Buccarello, Valentina Cesari and Ivan Toschi bred animals, administered taurine, expanded the colony and collected brains; Lucia Buccarello and Valentina Cesari performed behavioral tests; Stefania Cattaldo and Alessandro Mauro performed HPLC experiments; Silvia Michela Mazzola analyzed OFT video by Any Maze software; Luciano Calzari and Francesca Pregnolato, with help of Sara Guzzetti and Silvia Michela Mazzola, carried on statistical analyses; Luciano Calzari and Sara Guzzetti prepared all the figures and graphs; Silvia Russo collected, revised and discussed with all the Authors the data; Silvia Russo wrote the manuscript with the help of Luciano Calzari, Sara Guzzetti and Lucia Buccarello. All the authors reviewed the manuscript.

Conflicts of Interest: The authors declare no conflict of interest.

\section{Abbreviations}

$\begin{array}{ll}\text { AS } & \text { Angelman Syndrome } \\ \text { UBE3A } & \text { Ubiquitin-protein ligase E3A } \\ \text { GABA } & \text { Gamma aminobutyric acid } \\ \text { CNS } & \text { Central nervous system } \\ \text { OFT } & \text { Open field test } \\ \text { NORT } & \text { Novel object recognition test } \\ \text { PSD-95 } & \text { PostSynaptic Density-95 } \\ \text { pERK1/2 } & \text { Phospho-extracellular signal-regulated Kinase } \\ \text { ERK1/2 } & \text { Total-extracellular signal-regulated Kinase } \\ \text { GAPDH } & \text { Glyceraldehyde-3-phosphate dehydrogenase } \\ \text { HPLC } & \text { High performance liquid chromatography }\end{array}$

\section{Appendix A}

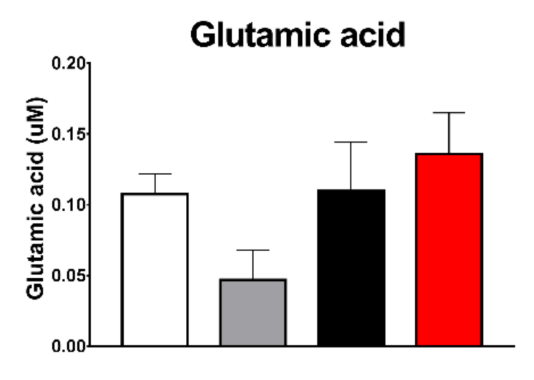

$$
\begin{aligned}
& \square m+/ p+\text { vehicle } \\
& \square \text { m+/p+ taurine } \\
& \text { m-/p+ vehicle } \\
& \square m-/ p+\text { taurine }
\end{aligned}
$$
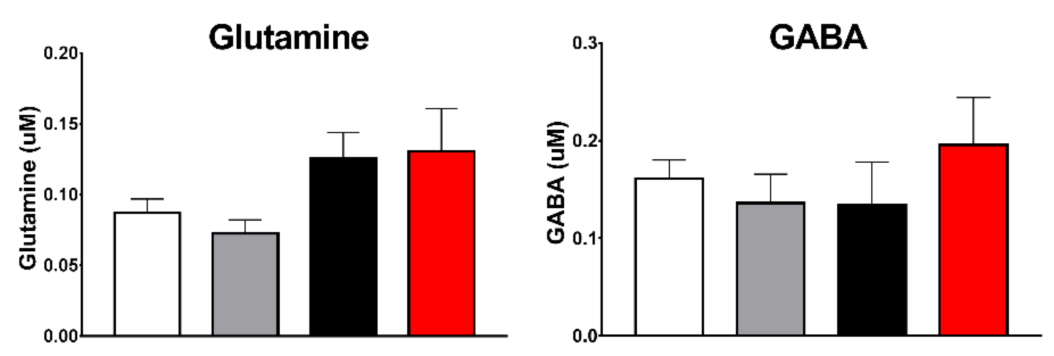

Figure A1. Measurements of neurotransmitters in whole brains evidenced no statistical differences between groups. HPLC experiments on whole mouse brain were carried out to quantify levels of Glutamic acid, Glutamine and Gamma AminoButyric Acid (GABA). Number of mouse brains: $\mathrm{wt}+$ vehicle $=13, \mathrm{wt}+$ taurine $=3, U b e 3 a^{m-/ p+}+$ vehicle $=5, U b e 3 a^{m-/ p+}+$ taurine $=6$. 


\section{References}

1. Williams, C.A.; Angelman, H.; Clayton-Smith, J.; Driscoll, D.J.; Hendrickson, J.E.; Knoll, J.H.; Magenis, R.E.; Schinzel, A.; Wagstaff, J.; Whidden, E.M.; et al. Angelman syndrome: Consensus for diagnostic criteria. Angelman Syndrome Foundation. Am. J. Med. Genet. 1995, 56, 237-238. [CrossRef] [PubMed]

2. Williams, C.A.; Beaudet, A.L.; Clayton-Smith, J.; Knoll, J.H.; Kyllerman, M.; Laan, L.A.; Magenis, R.E.; Moncla, A.; Schinzel, A.A.; Summers, J.A.; et al. Angelman syndrome 2005: Updated consensus for diagnostic criteria. Am. J. Med. Genet. Part A 2006, 140, 413-418. [CrossRef] [PubMed]

3. Burette, A.C.; Judson, M.C.; Burette, S.; Phend, K.D.; Philpot, B.D.; Weinberg, R.J. Subcellular organization of UBE3A in neurons. J. Comp. Neurol. 2017, 525, 233-251. [CrossRef] [PubMed]

4. Grier, M.D.; Carson, R.P.; Lagrange, A.H. Toward a broader view of Ube3a in a mouse model of Angelman syndrome: Expression in brain, spinal cord, sciatic nerve and glial cells. PLoS ONE 2015, 10, e0124649. [CrossRef] [PubMed]

5. Dagli, A.I.; Mueller, J.; Williams, C.A. Angelman syndrome. In Genereviews((r)); Adam, M.P., Ardinger, H.H., Pagon, R.A., Wallace, S.E., Bean, L.J.H., Stephens, K., Amemiya, A., Eds.; University of Washington: Seattle, WA, USA, 1993.

6. Margolis, S.S.; Sell, G.L.; Zbinden, M.A.; Bird, L.M. Angelman syndrome. Neurotherapeutics 2015, 12, 641-650. [CrossRef] [PubMed]

7. Roden, W.H.; Peugh, L.D.; Jansen, L.A. Altered GABA(A) receptor subunit expression and pharmacology in human Angelman syndrome cortex. Neurosci. Lett. 2010, 483, 167-172. [CrossRef] [PubMed]

8. Santini, E.; Turner, K.L.; Ramaraj, A.B.; Murphy, M.P.; Klann, E.; Kaphzan, H. Mitochondrial superoxide contributes to hippocampal synaptic dysfunction and memory deficits in angelman syndrome model mice. J. Neurosci. 2015, 35, 16213-16220. [CrossRef] [PubMed]

9. Llewellyn, K.J.; Nalbandian, A.; Gomez, A.; Wei, D.; Walker, N.; Kimonis, V.E. Administration of coq10 analogue ameliorates dysfunction of the mitochondrial respiratory chain in a mouse model of angelman syndrome. Neurobiol. Dis. 2015, 76, 77-86. [CrossRef] [PubMed]

10. Berkowitz, B.A.; Lenning, J.; Khetarpal, N.; Tran, C.; Wu, J.Y.; Berri, A.M.; Dernay, K.; Haacke, E.M.; Shafie-Khorassani, F.; Podolsky, R.H.; et al. In vivo imaging of prodromal hippocampus ca1 subfield oxidative stress in models of alzheimer disease and angelman syndrome. FASEB J. 2017, 31, 4179-4186. [CrossRef] [PubMed]

11. Judson, M.C.; Wallace, M.L.; Sidorov, M.S.; Burette, A.C.; Gu, B.; van Woerden, G.M.; King, I.F.; Han, J.E.; Zylka, M.J.; Elgersma, Y.; et al. GABAergic neuron-specific loss of Ube3a causes Angelman syndrome-like EEG abnormalities and enhances seizure susceptibility. Neuron 2016, 90, 56-69. [CrossRef] [PubMed]

12. Egawa, K.; Kitagawa, K.; Inoue, K.; Takayama, M.; Takayama, C.; Saitoh, S.; Kishino, T.; Kitagawa, M.; Fukuda, A. Decreased tonic inhibition in cerebellar granule cells causes motor dysfunction in a mouse model of Angelman syndrome. Sci. Transl. Med. 2012, 4. [CrossRef] [PubMed]

13. Benitez-Diaz, P.; Miranda-Contreras, L.; Mendoza-Briceno, R.V.; Pena-Contreras, Z.; Palacios-Pru, E. Prenatal and postnatal contents of amino acid neurotransmitters in mouse parietal cortex. Dev. Neurosci. 2003, 25, 366-374. [CrossRef] [PubMed]

14. Adedara, I.A.; Abolaji, A.O.; Idris, U.F.; Olabiyi, B.F.; Onibiyo, E.M.; Ojuade, T.D.; Farombi, E.O. Neuroprotective influence of taurine on fluoride-induced biochemical and behavioral deficits in rats. Chem. Biol. Interact. 2017, 261, 1-10. [CrossRef] [PubMed]

15. Kim, H.Y.; Kim, H.V.; Yoon, J.H.; Kang, B.R.; Cho, S.M.; Lee, S.; Kim, J.Y.; Kim, J.W.; Cho, Y.; Woo, J.; et al. Taurine in drinking water recovers learning and memory in the adult APP/PS1 mouse model of Alzheimer's disease. Sci. Rep. 2014, 4, 7467. [CrossRef] [PubMed]

16. Mezzomo, N.J.; Fontana, B.D.; Kalueff, A.V.; Barcellos, L.J.G.; Rosemberg, D.B. Understanding taurine CNS activity using alternative zebrafish models. Neurosci. Biobehav. Rev. 2017, 83, 525-539. [CrossRef] [PubMed]

17. Leon, R.; Wu, H.; Jin, Y.; Wei, J.; Buddhala, C.; Prentice, H.; Wu, J.Y. Protective function of taurine in glutamate-induced apoptosis in cultured neurons. J. Neurosci. Res. 2009, 87, 1185-1194. [CrossRef] [PubMed]

18. Gebara, E.; Udry, F.; Sultan, S.; Toni, N. Taurine increases hippocampal neurogenesis in aging mice. Stem Cell Res. 2015, 14, 369-379. [CrossRef] [PubMed]

19. Chesnoy-Marchais, D. Persistent GABAA/C responses to gabazine, taurine and $\beta$-alanine in rat hypoglossal motoneurons. Neuroscience 2016, 330, 191-204. [CrossRef] [PubMed] 
20. Reyes-Haro, D.; Cabrera-Ruiz, E.; Estrada-Mondragon, A.; Miledi, R.; Martinez-Torres, A. Modulation of GABA-A receptors of astrocytes and STC-1 cells by taurine structural analogs. Amino Acids 2014, 46, 2587-2593. [CrossRef] [PubMed]

21. Toyoda, A.; Iio, W. Antidepressant-like effect of chronic taurine administration and its hippocampal signal transduction in rats. Adv. Exp. Med. Biol. 2013, 775, 29-43. [CrossRef] [PubMed]

22. Terrill, J.R.; Pinniger, G.J.; Graves, J.A.; Grounds, M.D.; Arthur, P.G. Increasing taurine intake and taurine synthesis improves skeletal muscle function in the $\mathrm{mdx}$ mouse model for Duchenne Muscular Dystrophy. J. Physiol. 2016, 594, 3095-3110. [CrossRef] [PubMed]

23. Neuwirth, L.S.; Volpe, N.P.; Ng, S.; Marsillo, A.; Corwin, C.; Madan, N.; Ferraro, A.M.; El Idrissi, A. Taurine recovers mice emotional learning and memory disruptions associated with fragile $\mathrm{X}$ syndrome in context fear and auditory cued-conditioning. Adv. Exp. Med. Biol. 2015, 803, 425-438. [CrossRef] [PubMed]

24. Silva-Santos, S.; van Woerden, G.M.; Bruinsma, C.F.; Mientjes, E.; Jolfaei, M.A.; Distel, B.; Kushner, S.A.; Elgersma, Y. Ube3a reinstatement identifies distinct developmental windows in a murine Angelman syndrome model. J. Clin. Investig. 2015, 125, 2069-2076. [CrossRef] [PubMed]

25. Godavarthi, S.K.; Dey, P.; Maheshwari, M.; Jana, N.R. Defective glucocorticoid hormone receptor signaling leads to increased stress and anxiety in a mouse model of Angelman syndrome. Hum. Mol. Genet. 2012, 21, 1824-1834. [CrossRef] [PubMed]

26. Cao, C.; Rioult-Pedotti, M.S.; Migani, P.; Yu, C.J.; Tiwari, R.; Parang, K.; Spaller, M.R.; Goebel, D.J.; Marshall, J. Impairment of TrkB-PSD-95 signaling in Angelman syndrome. PLoS Biol. 2013, 11, e1001478. [CrossRef] [PubMed]

27. Aguilar-Martinez, E.; Morrisroe, C.; Sharrocks, A.D. The ubiquitin ligase UBE3A dampens ERK pathway signalling in HPV E6 transformed HeLa cells. PLoS ONE 2015, 10, e0119366. [CrossRef] [PubMed]

28. Stornetta, R.L.; Zhu, J.J. Ras and Rap signaling in synaptic plasticity and mental disorders. Neuroscientist 2011, 17, 54-78. [CrossRef] [PubMed]

29. Sved, D.W.; Godsey, J.L.; Ledyard, S.L.; Mahoney, A.P.; Stetson, P.L.; Ho, S.; Myers, N.R.; Resnis, P.; Renwick, A.G. Absorption, tissue distribution, metabolism and elimination of taurine given orally to rats. Amino Acids 2007, 32, 459-466. [CrossRef] [PubMed]

30. Kovacs, A.D.; Pearce, D.A. Location- and sex-specific differences in weight and motor coordination in two commonly used mouse strains. Sci. Rep. 2013, 3, 2116. [CrossRef] [PubMed]

31. Hernandez-Benitez, R.; Ramos-Mandujano, G.; Pasantes-Morales, H. Taurine stimulates proliferation and promotes neurogenesis of mouse adult cultured neural stem/progenitor cells. Stem Cell Res. 2012, 9, 24-34. [CrossRef] [PubMed]

32. El Idrissi, A. Taurine increases mitochondrial buffering of calcium: Role in neuroprotection. Amino Acids 2008, 34, 321-328. [CrossRef] [PubMed]

33. Prentice, H.; Pan, C.; Gharibani, P.M.; Ma, Z.; Price, A.L.; Giraldo, G.S.; Retz, H.M.; Gupta, A.; Chen, P.C.; Chiu, H.; et al. Analysis of neuroprotection by taurine and taurine combinations in primary neuronal cultures and in neuronal cell lines exposed to glutamate excitotoxicity and to hypoxia/re-oxygenation. Adv. Exp. Med. Biol. 2017, 975, 207-216. [CrossRef] [PubMed]

34. Wu, J.Y.; Wu, H.; Jin, Y.; Wei, J.; Sha, D.; Prentice, H.; Lee, H.H.; Lin, C.H.; Lee, Y.H.; Yang, L.L. Mechanism of neuroprotective function of taurine. Adv. Exp. Med. Biol. 2009, 643, 169-179. [CrossRef] [PubMed]

35. El Idrissi, A.; Neuwirth, L.S.; L'Amoreaux, W. Taurine regulation of short term synaptic plasticity in fragile $\mathrm{X}$ mice. J. Biomed. Sci. 2010, 17, S15. [CrossRef] [PubMed]

36. Kilb, W.; Kirischuk, S.; Luhmann, H.J. Role of tonic GABAergic currents during pre- and early postnatal rodent development. Front. Neural Circuits 2013, 7, 139. [CrossRef] [PubMed]

37. Wang, B.; Gao, Y.; Xiao, Z.; Chen, B.; Han, J.; Zhang, J.; Wang, X.; Dai, J. Erk1/2 promotes proliferation and inhibits neuronal differentiation of neural stem cells. Neurosci. Lett. 2009, 461, 252-257. [CrossRef] [PubMed]

38. Terrill, J.R.; Pinniger, G.J.; Nair, K.V.; Grounds, M.D.; Arthur, P.G. Beneficial effects of high dose taurine treatment in juvenile dystrophic mdx mice are offset by growth restriction. PLoS ONE 2017, 12, e0187317. [CrossRef] [PubMed]

39. Jiang, Y.H.; Armstrong, D.; Albrecht, U.; Atkins, C.M.; Noebels, J.L.; Eichele, G.; Sweatt, J.D.; Beaudet, A.L. Mutation of the Angelman ubiquitin ligase in mice causes increased cytoplasmic p53 and deficits of contextual learning and long-term potentiation. Neuron 1998, 21, 799-811. [CrossRef] 
40. Seibenhener, M.L.; Wooten, M.C. Use of the open field maze to measure locomotor and anxiety-like behavior in mice. J. Vis. Exp. 2015. [CrossRef] [PubMed]

41. Ennaceur, A.; Delacour, J. A new one-trial test for neurobiological studies of memory in rats. 1: Behavioral data. Behav. Brain Res. 1988, 31, 47-59. [CrossRef]

42. Antunes, M.; Biala, G. The novel object recognition memory: Neurobiology, test procedure, and its modifications. Cogn. Process. 2012, 13, 93-110. [CrossRef] [PubMed]

43. Perucho, J.; Gonzalo-Gobernado, R.; Bazan, E.; Casarejos, M.J.; Jimenez-Escrig, A.; Asensio, M.J.; Herranz, A.S. Optimal excitation and emission wavelengths to analyze amino acids and optimize neurotransmitters quantification using precolumn opa-derivatization by hplc. Amino Acids 2015, 47, 963-973. [CrossRef] [PubMed]

44. Sanli, N.; Tague, S.E.; Lunte, C. Analysis of amino acid neurotransmitters from rat and mouse spinal cords by liquid chromatography with fluorescence detection. J. Pharm. Biomed. Anal. 2015, 107, 217-222. [CrossRef] [PubMed]

45. Zeger, S.L.; Liang, K.Y.; Albert, P.S. Models for longitudinal data: A generalized estimating equation approach. Biometrics 1988, 44, 1049-1060. [CrossRef] [PubMed]

(C) 2018 by the authors. Licensee MDPI, Basel, Switzerland. This article is an open access article distributed under the terms and conditions of the Creative Commons Attribution (CC BY) license (http:/ / creativecommons.org/licenses/by/4.0/). 